\title{
Leadership \& Professional Development: Specialty Silos in Medicine
}

\author{
Zahir Kanjee, MD, MPH',2*, Leslie Bilello, MD2,3
}

${ }^{1}$ Department of Medicine, Beth Israel Deaconess Medical Center, Boston, Massachusetts; ${ }^{2}$ Harvard Medical School, Boston, Massachusetts; ${ }^{3}$ Department of Emergency Medicine, Beth Israel Deaconess Medical Center, Boston, Massachusetts.

Siloed, adj.:

Kept in isolation in a way that hinders communication and cooperation ...

\section{—Merriam-Webster's Dictionary}

umans naturally separate into groups, and the medical field is no exception. Being a member of a likeminded group, such as one's specialty, can improve self-esteem and provide social organization: it feels good to identify with people we admire. Through culture, these specialty-based groups implicitly and explicitly guide and encourage positive attributes or behaviors like a hospitalist's thoroughness or an emergency medicine physician's steady management of unstable patients. Our specialties also provide support and understanding in challenging times.

Despite these positive aspects, such divisions can negatively affect interprofessional relationships when our specialties become siloed. A potential side-effect of building up ourselves and our own groups is that we can implicitly put others down. For example, a hospitalist who spends extra time on the phone regularly updating each patient's family will appropriately take pride in their practice, but over time this can also lead to an unreasonable assumption that physicians in other departments with different routines are not as committed to outstanding communication.

These rigid separations facilitate the fundamental attribution error, the tendency to ascribe a problem or disagreement to a colleague's substandard character or ability. Imagine that the aforementioned hospitalist's phone call delays a response to an admission page from the emergency room. The emergency medicine physician, who is waiting to sign out the admission while simultaneously managing many sick and complex patients, could assume the hospitalist is being disrespectful, rather than also working hard to provide the best care. Our siloed specialty identities can lead us to imagine the worst in each other and exacerbate intergroup conflict. ${ }^{1}$

Silos in medicine also adversely affect patients. Poor communication and lack of information-sharing across disciplines can lead to medical error ${ }^{2}$ and stifle dissemination of safer practices. $^{3}$ Further, the unintentional disparaging of other

*Corresponding Author: Zahir Kanjee, MD, MPH; Email: zkanjee@bidmc. harvard.edu; Telephone: 617-754-4677; Twitter: @zahirkanjee.

Published online first May 21, 2021

Received: May 2, 2021; Accepted: May 10, 2021

(c) 2021 Society of Hospital Medicine DOI 10.12788/jhm.3647 medical specialties undermines the confidence our patients have in all of us; a patient within earshot of the hospitalist expressing annoyance at the "impatient" emergency medicine physician who "won't stop paging," or the emergency medicine physician complaining about the hospitalist who "refuses to call back," will lose trust in each of their providers.

We suggest three steps to reduce the negative impact of specialty silos in medicine:

1. Get to know each other personally. Friendly conversation during work hours and social interaction outside the hospital can inoculate against interspecialty conflict by putting a human face on our colleagues. The resultant relationships make it easier to work together and see things from another's perspective.

2. Emphasize our shared affiliations. ${ }^{4}$ The greater the salience of a mutual identity as "healthcare providers," the more likely we are to recognize each other's unique contributions and question the stereotypes we imagine about one another.

3. Consider projects across specialties. Interdepartmental data-sharing and joint meetings, including educational conferences, can facilitate situational awareness, synergy, and efficient problem-solving.

Our medical specialties will continue to group together. While these groups can be a source of strength and meaning, silos can interfere with professional alliances and effective patient care. Mitigating the harmful effects of silos can benefit all of us and our patients.

Authors' note: This article was previously published using the term "tribalism," which we have since learned is derogatory to Indigenous Americans and others. We apologize for any harm. We have retracted and republished the article without this language. We appreciate readers teaching us how to choose better words so all people feel respected and valued.

Disclosures: The authors have no conflicts to disclose.

\section{References}

1. Fiol CM, Pratt MG, O'Connor EJ. Managing intractable identity conflicts. Acad Management Rev. 2009;34(1):32-55. https://doi.org/10.5465/ amr.2009.35713276

2. Horowitz LI, Meredith T, Schuur JD, et al. Dropping the baton: a qualitative analysis of failures during the transition from emergency department to inpatient care. Ann Emerg Med. 2009;53(6): 701-710. https://doi.org/ 10.1016/ j.annemergmed.2008.05.007

3. Paine, LA, Baker DR, Rosenstein B, Pronovost PJ. The Johns Hopkins Hospital: identifying and addressing risks and safety issues. JT Comm J Qual Saf. 2004;30(10):543-550. https://doi.org/10.1016/s1549-3741(04)30064-x

4. Burford B. Group processes in medical education: learning from social identity theory. Med Educ. 2012;46(2):143-152. https://doi.org/10.1111/j.13652923.2011.04099.x 